\title{
Evaluation of Improvement in Clinical and Left Ventricular Systolic Functions after Cardiac Resynchronization Therapy in Patients with Heart Failure in the Indian Population
}

\author{
Shaikh Saiful Haque Zahed ${ }^{1}$, Naushi Mujeeb²*
}

\section{Shaikh Saiful Haque Zahed', Naushi Mujeeb $^{2 *}$}

'Department of Medicine and Consultant Cardiology, Central Referral Hospital, Sikkim Manipal Institute of Medical Sciences, SMU, Gangtok, Sikkim, INDIA. ${ }^{2}$ Department of Physiology, Sikkim Manipal Institute of Medical Sciences, SMU, Gangtok, Sikkim, INDIA.

\section{Correspondence}

\section{Dr. Naushi Mujeeb}

Associate Professor, Department of Physiology, Sikkim Manipal Institute of Medical Sciences, SMU, Gangtok, Sikkim, INDIA.

Phone: +91 7007022493

Email: naushimujeeb78@gmail.com

\section{History}

- Submission Date: 05-01-2021

- Review completed: 22-02-2021.

- Accepted Date: 14-03-2021.

DOI : 10.5530/ijcep.2021.8.1.8

\section{Article Available online} http://www.ijcep.org/v8/i1

\section{Copyright}

(C) 2021 Phcog.Net. This is an openaccess article distributed under the terms of the Creative Commons Attribution 4.0 International license.

\begin{abstract}
Background and Aim: Heart Failure (HF) is a chronic progressive disease associated with substantial morbidity and mortality. It is a major and growing global public health problem affecting millions of people worldwide. Despite optimal pharmacological therapy, many patients may still have significant symptoms affecting their functional capacity and quality of life. This study was conducted to determine the efficacy of Cardiac Resynchronization Therapy (CRT) in improving clinical and systolic echocardiographic parameters of the Left Ventricle in patients with heart failure in the Indian population. Methods: This is a non-randomized prospective observational study conducted at the Department of Cardiology, Fortis Escorts Heart Institute, New Delhi, from $1^{\text {st }}$ September 2014-31 $1^{\text {st }}$ December 2015. A total of 70 cases eligible for CRT based on inclusion and exclusion criteria were included in the study. Echocardiographic parameters for Left ventricular systolic functions were measured pre CRT implantation, 3 months and 6 months post CRT implantation. Data were analyzed using standard statistical tests. Results: Present study showed statistically significant improved values $(P \leq 0.0001)$ for the clinical response measured in terms of New York Heart Association (NYHA) class and Echocardiographic response measured by Left Ventricular systolic functions (LVEF, LVESV, and LVESDs) in patients treated with CRT, measured at 3 months and 6months post CRT implantation. Conclusion: This study supports the efficacy of CRT for the treatment of Heart Failure in the population studied in terms of improving patient's clinical and echocardiographic Left Ventricular Systolic Functions.

Key words: Cardiac Resynchronization Therapy, Heart Failure, NYHA class, Left ventricular ejection fraction, Left ventricular end systolic volume, Left ventricular end systolic diameter.
\end{abstract}

\section{INTRODUCTION}

Heart failure is a syndrome in which the patients have the following features: typically, shortness of breath at rest or during exertion, fatigue, signs of fluid retention such as pulmonary congestion or ankle swelling, and evidence of an abnormality of the structure or function of the heart at rest.

In the European countries with a total population of $>900$ million, there are at least 15 million patients with $\mathrm{HF}^{[1]}$ The burden of Heart Failure in developing countries is also quite alarming. Huffman and Dorairaj have estimated the prevalence of heart failure in India in the range of 1.3 to 4.6 million. ${ }^{[2]}$

Cardiac Resynchronization Therapy (CRT) is a wellestablished treatment for patients of heart failure with an impaired LV function and a wide QRS complex on Electro Cardiography (ECG). CRT that aims to resolve electromechanical dyssynchrony has become an established mode of therapy among a selective group of patients with symptomatic NYHA Class II/III and ambulatory class IVA systolic heart failure. ${ }^{[3,4]}$ This therapy has revolutionized the

treatment of patients with heart failure whose only previous option was a cardiac transplant.

There is a wealth of data on the improvement of the clinical and systolic parameters in literature, but very limited data is available in Heart failure patients who receive this therapy in the Indian population.

Several studies have provided strong evidence supporting the benefits of CRT in patients with HF.

The MIRACLE (Multicenter InSync Randomized Clinical Evaluation) trial was conducted between 1998 and 2000 to evaluate the benefits of CRT. ${ }^{[5]}$ This trial included 453 patients with moderate to severe symptoms of heart failure. When compared with the control group, patients randomly assigned to CRT group demonstrated a significant improvement in quality-of-life score, NYHA functional class, peak VO2, treadmill exercise time, and LVEF. This study also showed that fewer patients from the CRT group needed hospitalization ( $8 \%$ versus $15 \%$ ) or intravenous medications ( $7 \%$ versus 15\%) for the worsening of heart failure (both $\mathrm{P}<0.05$ ).

Cite this article: Zahed SKSH, Mujeeb N. Evaluation of Improvement in Clinical and Left Ventricular Systolic Functions after Cardiac Resynchronization Therapy in Patients with Heart Failure in the Indian Population. Int J Clin Exp Physiol. 2021;8(1):31-4. 
COMPANION (Comparison of Medical Therapy, Pacing, and Defibrillation in Heart Failure) study in the year 2000 was a clinical trial for comparing drug therapy alone with drug therapy in combination with cardiac resynchronization in patients. ${ }^{[6]}$ The combined endpoint of mortality or hospitalization for heart failure was reduced by $40 \%$ in patients receiving CRT-D and 35\% in patients receiving CRT alone (both $\mathrm{P}<0.001)$ compared with optimal medical therapy alone.

The COMPANION trial supported the results of earlier trials of resynchronization therapy in improving symptoms, exercise tolerance, and quality of life in the selective group of heart failure patients with ventricular dyssynchrony.

The following randomized controlled trials- REVERSE (Resynchronization Reverses Remodeling in Systolic Left Ventricular Dysfunction) trial, ${ }^{[7]}$ MADIT-CRT (Multicenter Automatic Defibrillator Implantation Trial with Cardiac Resynchronization Therapy) ${ }^{[8]}$ and RAFT (Resynchronization/defibrillation for Ambulatory Heart Failure Trial) ${ }^{[9]}$ are considered to be among the landmark studies of CRT in the patient population.

\section{MATERIALS AND METHODS}

The present study is a single-center, hospital-based non-randomized prospective observational study conducted at the Department of Cardiology, Fortis Escorts Heart Institute, New Delhi, from $1^{\text {st }}$ September 2014-31 $1^{\text {st }}$ December 2015 (1 year and 3 months).

\section{Study Population}

All adult patients admitted to Fortis Escorts Heart Institute, New Delhi, during the study period for first CRT implantation and satisfying the enrolment criteria of the study.

\section{Inclusion Criteria}

1. Patients willing for CRT device implantation for acceptable indications of HF.

a) Patients of Heart Failure with NYNA Class II, III / Ambulatory IV (IVA) despite optimal medical therapy.

b) Left Ventricular Systolic dysfunction, LVEF $\leq 35 \%$

c) Wide QRS complex $\geq 120$ msec.

2. Patient willing to participate in the study.

3. Willing for follow-up.

\section{Exclusion Criteria}

1. Not willing to participate.

2. Moribund patient

3. Sever multi-organ dysfunction.

4. Non-ambulatory patients.

5. Acute coronary syndrome (less than 3 months)

6. Recent coronary revascularization (during the last 3 months)

7. Patients in persistent AF

8. Treatment-resistant hypertension

9. Severe obstructive pulmonary disease.

10. Reduced life expectancy not associated with cardiovascular disease (less than one year).

\section{Methodology}

Total 70 cases eligible for CRT were enrolled and were evaluated as follows:

Pre-intervention assessment done.

Inclusion/Exclusion Criteria applied.
Echocardiographic assessment- for Left Ventricular Systolic Function.

Three cases were excluded from the study who couldn't come for follow-up. Intervention: CRT device (CRT-P or CRT-D) implantation.

Approval for the study was taken from the Ethics Committee. Informed consent was taken from all the study participants to participate in the study. The investigator also signed a confidentiality statement on the informed consent before recruitment. A detailed history and clinical examinations were done and recorded on a predesigned proforma. Clinical parameters of breathlessness were evaluated by NYHA classification $^{[10]}$ at baseline and follow-up. ECG was done at baseline and during follow-up.

The subjects were advised to follow-up at 3 and 6 months after implantation. They were free to report sooner in case of worsening of symptoms or any other complaints. At follow-up, clinical and echocardiographic study parameters were recorded.

Echocardiographic parameters were recorded on the Phillips i33 echocardiography machine by experienced operators. Parameters recorded at baseline were Left Ventricular Ejection Fraction (LVEF in $\%)$, Left Ventricular End Systolic Diameter (LVESD in millimeters mm), Left Ventricular End Systolic Volume (LVESV in cubic mm).

\section{Measured Outcome Variables were}

a) Clinical Responder: Percentage of patients with improvement of at least 1 NYHA Class.

b) Echo Responder: Percentage of patients with improvement in LVEF of at least $5 \%$.

c) Reverse Remodelling Responder: Percentage of patients with a decrease in LVESV of at least $15 \%$.

\section{Statistical Analysis of Data}

Data analysis and statistics were done with the help of IBM ${ }^{\circ}$ SPSS Statistics version 20.0 for windows.

Categorical variables are expressed as numbers and percentages; continuous variables are expressed as mean \pm standard deviations.

A comparison of the clinical and echocardiographic parameters was done prior to and after CRT was performed using paired and unpaired Student t-test and Pearson correlations as appropriate. P-value of $<0.05$ was taken as statistically significant.

\section{RESULTS}

All the patients in this study receiving CRT showed improvement in clinical and echo systolic parameters. In the present study, after CRT implantation at 3 and 6 months follow-up, there was an improvement by at least 1 NYHA class in $76.1 \%$ and $77.6 \%$ subjects, respectively. There was a statically significant improvement in LVEF, LVESV, and LVESD post CRT implantation.

Figure 1 shows the sex distribution of the subjects in the study.

The mean age of the subjects in the study group was $62.5 \pm 11.7$ years. Gender distribution Male : Female gender ratio of the enrolled subjects was approximately $4: 1, n=54(80.5 \%)$ for males and $n=13(19.5 \%)$ for females.

Figure 2 shows the NYHA class distribution in subjects pre-treatment. In this study, before CRT device implantation majority of the cases were in NYHA functional class III $(\mathrm{n}=39,58.2 \%)$. However, a substantial number of cases were in ambulatory NYHA functional class IVA ( $\mathrm{n}=$ 28; $41.8 \%)$. The mean preimplant NYHA class of the study was $3.42 \pm$ 0.497 NYHA class.

Table 1 shows clinical response by NYHA class.

Table 2 shows LVEF values at baseline and follow-up. 


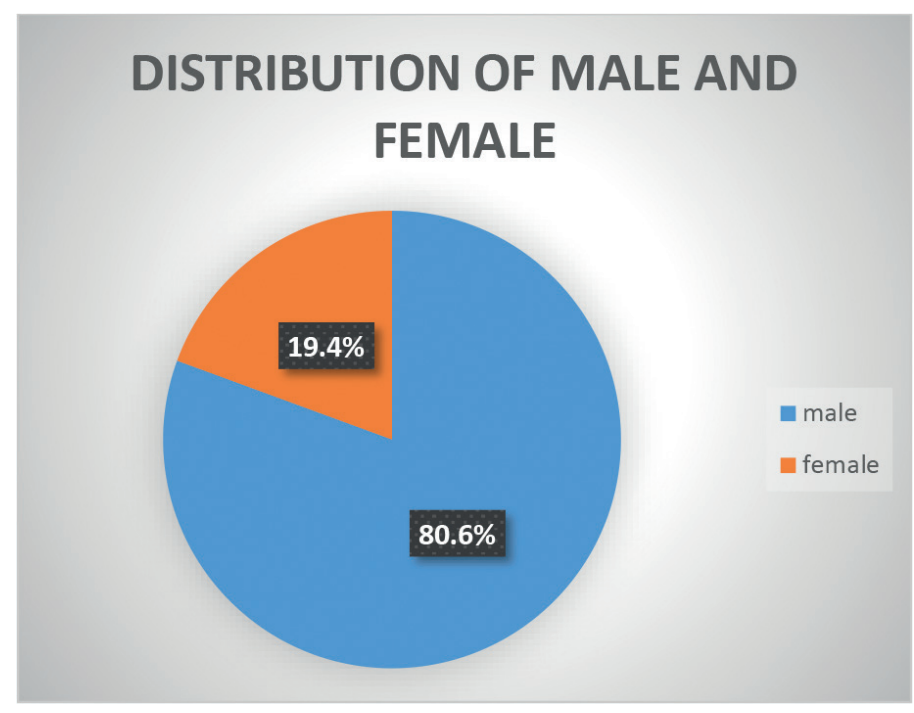

Figure 1: Sex distribution of the subjects in the study.

\section{NYHA CLASS DISTRIBUTION}

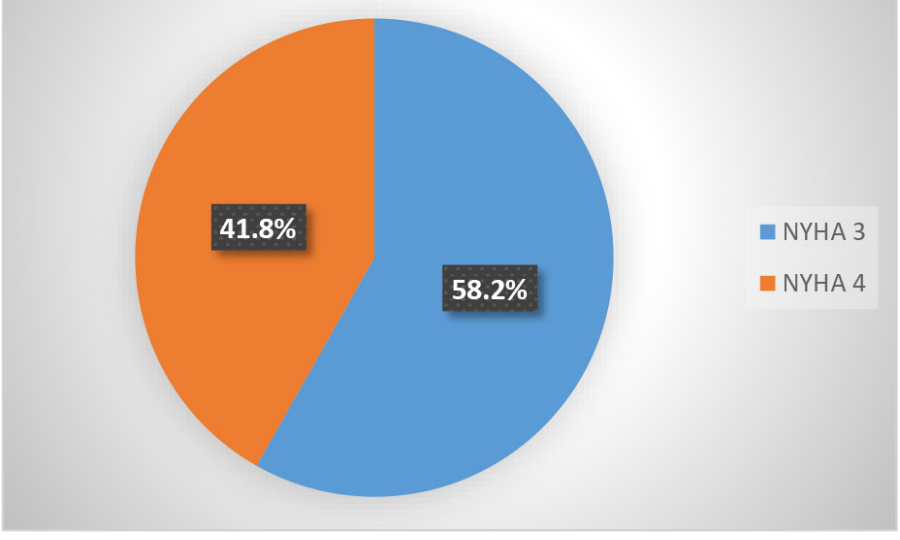

Figure 2: NYHA class pre-treatment.

Table 1: Clinical response- NYHA class improvement at 3 and 6 months.

$\begin{array}{lcccc} & \mathrm{n} & \% & \text { Mean } & \mathrm{P} \\ \text { Response at 3 months } & 51 / 67 & 76.10 \% & 2.57 \pm 0.52 & 0.0001 \\ \text { Response at 6 months } & 52 / 67 & 77.60 \% & 2.31 \pm 0.56 & 0.0001\end{array}$

Table 3 shows LVESV at baseline and follow-up.

Table 4 shows LVESD at baseline and follow-up.

\section{DISCUSSION}

Before the era of modern treatment of heart failure, which began in 1990 , $60 \%-70 \%$ of people died within 5 years. An epidemic of hospitalization for Heart Failure in many countries was seen in this era because of frequent and recurrent hospitalization for worsening of symptoms of heart failure. With the advent of new medical therapy in the form of beta-blockers, angiotensin-converting enzyme inhibitors or angiotensin receptor blockers, mineralocorticoid antagonists, and diuretics, in the past $15-20$ years, mortality has been reduced by $30-40 \%$. However, many patients, despite the use of optimal pharmacologic therapy, may still have significant symptoms affecting their functional capacity and quality of life. Cardiac Resynchronization Therapy (CRT) has been the newest
Table 2: LVEF values at baseline and follow up.

$\begin{array}{lcccc} & \text { Mean } & \text { SD } & \begin{array}{c}\text { Mean } \\ \text { Difference }\end{array} & \text { P } \\ \text { Baseline } & 24.52 & 5.5 & & \\ 3 \text { months } & 28.9 & 6.4 & 4.4 \pm 3.7 & 0.0001 \\ 6 \text { months } & 31.2 & 8.3 & 6.7 \pm 5.9 & 0.0001\end{array}$

Table 3: LVESV value at baseline and follow up.

\begin{tabular}{|cccc|}
\hline & Mean & SD & P \\
\hline Baseline LVESV & 131.75 & 18.81 & \\
\hline LVESV 3 months & 112.88 & 19.6 & 0.001 \\
LVESV 6 months & 102.72 & 21.67 & 0.001 \\
\hline
\end{tabular}

SD: Standard Deviation

Table 4: LVESD baseline and follow up.

\begin{tabular}{|ccccc|} 
& Mean & SD & $\begin{array}{c}\text { Mean } \\
\text { Difference }\end{array}$ & P \\
\hline Baseline & 50.51 & 9.546 & & \\
LVESD at 3 months & 44.49 & 9.55 & $6 \pm 4.5$ & 0.0001 \\
LVESD at 6 months & 42.36 & 10.172 & $8.1 \pm 6.5$ & 0.0001 \\
\hline
\end{tabular}

addition to the armamentarium of HF therapies based on strong evidence from well-designed clinical trials. ${ }^{[11]}$

Clinical outcomes measures after CRT implantation that have been evaluated in major clinical trials include NYHA functional class, quality of life, objective measures of exercise capacity such as the 6-min walk test, exercise duration, and VO2 max.

The present study is a single-center, hospital-based non-randomized prospective observational study to measure the effect of CRT on the echocardiographic systolic functions of the heart. The study was conducted in the Department of Cardiology, Fortis Escorts Heart Institute, New Delhi.

Using the pre-specified inclusion and exclusion criteria a total of 67 cases were included in the study for final analysis. The total study duration was of 1 year and 3 months with follow-up at 3 and 6 months.

In the present study after CRT implantation at 3 and 6 months follow-up, there was an improvement by at least 1 class (CRT Response) in $76.1 \%$ and $77.6 \%$ subjects, respectively. Pre-implant all subjects were in NYHA class III $(n=39,58.2 \%)$ or IVA $(n=28 ; 41.8 \%)$, whereas at mean follow-up, 44 subjects $(65.6 \%)$ were either in NYHA class I or II at 6 months. CARE$\mathrm{HF}^{[12]}$ has also shown a mean NYHA class improvement of 0.6 (CI $0.4-0.7$ ) NYHA classes. The CRT response rate of the present study is slightly better than results from other RCTs such as MUSTIC, ${ }^{[13]}$ MUSTIC-AF, ${ }^{[14]}$ MIRACLE,${ }^{[5]}$ MIRACLE-ICD,${ }^{[15]}$ MIRACLE-ICD II, ${ }^{[16]}$ COMPANION,${ }^{[6]}$ CONTAK CD, ${ }^{[17]}$ and PATH-CHF. ${ }^{[18]}$ This is probably because in this study, most of the patients had QRS $\geq 150 \mathrm{~ms}$, had LBBB on ECG indicating dyssynchrony, and benefitted from the therapy.

Number and percentage of patients who showed an improvement in LVEF of $\geq 5 \%$ was $37 / 67(55.2 \%)$ at 3 months which improved to $42 / 67(62.6 \%)$ at 6 months of follow-up. Mean baseline LVEF was $24.52 \pm 5.503$, which improved to $28.9 \pm 6.4$ at 3 months with $\mathrm{P}=0.0001$ and to $31.2 \pm 8.3$ at 6 months with $\mathrm{P}=0.0001$. When LVEF at 3 and 6 months were compared, then also it was statistically significant $(\mathrm{P}=0.0001)$. The mean difference in LVEF at 3 month and 6 months was $-4.4 \pm 3.7$ and $-6.7 \pm 5.9$, respectively. MIRACLE,${ }^{[5]}$ CARE-HF, ${ }^{[12]}$ CONTAK CD, ${ }^{[17]}$ and MIRACLE ICD II ${ }^{[16]}$ studies also reported similar improvement in LVEF post CRT implantation. 
Baseline LVESV decreased significantly in this study at 3 months $(\mathrm{P}=0.0001)$ mean difference $18.8 \pm 10.5$ and at 6 months $(\mathrm{P}=0.0001)$ mean difference of $29.0 \pm 16 \mathrm{ml}$ when compared to baseline, there was a continued improvement between 3 and 6 months, the similar outcome in LVESV was reported in MIRACLE ${ }^{[5]}$ study.

Baseline LVESD was $50.51 \pm 9.546$ at baseline. This decreased at 3 months to $44.5 \pm 9.5$ with a difference in mean of $6.0 \pm 4.6(\mathrm{P}=0.0001)$. At 6 months, this further decreased to $42.3 \pm 10.1$ with a difference in mean of $8.1 \pm 6.5(\mathrm{P}=0.0001)$. Thus, the mean change in LVESD in this study was$6 \pm 4.5 \mathrm{~mm}$ at 3 months and $-8.1 \pm 6.5 \mathrm{~mm}$ at 6 months. A similar decrease in LVESD was seen in the MIRACLE study, the study by CONTAK, and by Gasparini et al..$^{[19]}$

\section{Strengths and Limitations of the Study}

1. It is not a multicenter study.

2. The current study is an observational and uncontrolled investigation and the low sample size might interfere with the results, though statistical sample size calculation showed that in this study, the sample size is enough and adequately powered to show differences between the measurements before and after CRT.

\section{CONCLUSION}

In the present era, CRT has proven to be a lifesaving therapy for a selected group of heart failure patients, backed by a wealth of data from well-conducted randomized clinical trials demonstrating its effect on morbidity and mortality.

However, as with any therapeutic modality, CRT also has its limitations. Despite stringent inclusion criteria, the nonresponder rate is still around $30 \%$. There is robust data from well-organized randomized controlled trials on the efficacy of CRT, demonstrating its effect on echocardiographic LV systolic function and functional outcome.

This study has reported similar results in the study population, concluding that CRT appears promising for the treatment of HF. Thus, this study supports the efficacy of CRT in the population studied in terms of improving a patient's clinical symptoms, LV reverse remodelling, and improvement in LVEF.

\section{ACKNOWLEDGEMENT}

We acknowledge the support provided to us by the Department of Cardiology, Fortis Escorts Heart Institute, New Delhi, where the study was conducted. We are also thankful to all the subjects who participated and cooperated in this study.

\section{CONFLICT OF INTEREST}

The authors declare no conflict of interest.

\section{ABBREVIATIONS}

CRT-P: Cardiac Resynchronization Therapy Pacemaker; CRT-D: Cardiac Resynchronization Therapy Defibrillator; NYHA: New York Heart Association; LVEF: Left Ventricular Ejection Fraction; LVESV: Left Ventricular End Systolic Volume; LVESD: Left Ventricular End Systolic Diameter; VO2 max: Maximum Oxygen Uptake.

\section{REFERENCES}

1. Dickstein K, Cohen-Solal A, Filippatos G, McMurray JJ, Ponikowski P, PooleWilson PA, et al. ESC guidelines for the diagnosis and treatment of acute and chronic heart failure 2008: The Task Force for the diagnosis and treatment of acute and chronic heart failure 2008 of the European Society of Cardiology. Developed in collaboration with the Heart Failure Association of the ESC (HFA) and endorsed by the European Society of Intensive Care Medicine (ESICM). Eur J Heart Fail. 2008;10(10):933-89.

2. Huffman MD, Prabhakaran D. Heart failure: Epidemiology and prevention in India. Natl Med J India. 2010;23(5):283-8.

3. Russo AM, Stainback RF, Bailey SR, Epstein AE, Heidenreich PA, Jessup M, et al. ACCF/HRS/AHA/ASE/HFSA/SCAI/SCCT/SCMR 2013 appropriate use criteria for implantable cardioverter-defibrillators and cardiac resynchronization therapy: A report of the American College of Cardiology Foundation appropriate 79 se criteria task force, Heart Rhythm Society, American Heart Association, American Society of Echocardiography, Heart Failure Society of America, Society for Cardiovascular Angiography and Interventions, Society of Cardiovascular Computed Tomography, and Society for Cardiovascular Magnetic Resonance. J Am Coll Cardiol. 2013;61(12):1318-68.

4. Epstein AE, DiMarco JP, Ellenbogen KA, Estes NA, Freedman RA, Gettes LS, et al. 2012 ACCF/AHA/HRS focused update incorporated into the ACCF/AHA/ HRS 2008 guidelines for device-based therapy of cardiac rhythm abnormalities: A report of the American College of Cardiology Foundation/American Heart Association Task Force on Practice Guidelines and the Heart Rhythm Society. J Am Coll Cardiol. 2013; 61(3):e6-75.

5. Abraham WT, Fisher WG, Smith AL, Delurgio DB, Leon AR, Loh E, et al. Cardiac resynchronization in chronic heart failure. N Engl J Med. 2002;346(24):1845-53.

6. Bristow MR, Saxon LA, Boehmer J, Krueger S, Kass DA, De Marco T, et al. Cardiac resynchronization therapy with or without an implantable defibrillator in advanced chronic heart failure. N Engl J Med. 2004;350(21):2140-50

7. Linde C, Abraham WT, Gold MR, Sutton MJ, Ghio S, Daubert C, et al. Randomized trial of cardiac resynchronization in mildly symptomatic heart failure patients and asymptomatic patients with left ventricular dysfunction and previous heart failure symptoms. J Am Coll Cardiol. 2008;52(23):1834-43.

8. Moss AJ, Hall WJ, Cannom DS, Klein H, Brown MW, Daubert JP, et al. Cardiac resynchronization therapy for the prevention of heart-failure events. $\mathrm{N}$ Engl J Med. 2009;361(14):1329-38

9. Tang AS, Wells GA, Talajic M, Arnold MO, Sheldon R, Connolly S, et al. Cardiac resynchronization therapy for mild-to-moderate heart failure. N Engl J Med. 2010;363(25):2385-95.

10. New York Heart Association Criteria Committee. Nomenclature and criteria for diagnosis of diseases of the heart and great vessels. $9^{\text {th }}$ ed. Boston Little Brown; 1994;334.

11. Khan GN, Curtis AB. Do the Official Guidelines for Cardiac Resynchronization Therapy Need to Be Changed? Devices for Cardiac Resynchronization: Technologic and Clinical Aspects. Boston, MA: Springer Science+Business Media, LLC; 2008;3-15

12. Cleland JG, Daubert JC, Erdmann E, Freemantle N, Gras D, Kappenberger L, et al. The effect of cardiac resynchronization on morbidity and mortality in heart failure. N Engl J Med. 2005;352(15):1539-49.

13. Cazeau S, Leclercq C, Lavergne T, Walker S, Varma C, Linde C, et al. Effects of multisite biventricular pacing in patients with heart failure and intraventricular conduction delay. N Engl J Med. 2001;344(12):873-80.

14. Linde C, Leclercq C, Rex S, Garrigue S, Lavergne T, Cazeau S, et al. Long-term benefits of biventricular pacing in congestive heart failure: Results from the Multisite STimulation in cardiomyopathy (MUSTIC) study. J Am Coll Cardiol. 2002;40(1):111-8.

15. Young JB, Abraham WT, Smith AL, Leon AR, Lieberman $R$, Wilkoff $B$, et al. Combined cardiac resynchronization and implantable cardioversion defibrillation in advanced chronic heart failure: The MIRACLE ICD Trial. JAMA 2003;289(20):2685-94.

16. Abraham WT, Young JB, Leon AR, Adler S, Bank AJ, Hall SA, et al. Effects of cardiac resynchronization on disease progression in patients with left ventricular systolic dysfunction, an indication for an implantable cardioverter-defibrillator and mildly symptomatic chronic heart failure. Circulation. 2004;110(18):2864-8.

17. Higgins SL, Hummel JD, Niazi IK, Giudici MC, Worley SJ, Saxon LA, et al. Cardiac resynchronization therapy for the treatment of heart failure in patients 88 with intraventricular conduction delay and malignant ventricular tachyarrhythmias. $J$ Am Coll Cardiol. 2003;42(8):1454-9.

18. Auricchio A, Stellbrink C, Sack S, Block M, Vogt J, Bakker P, et al. Long-term clinical effect of hemodynamically optimized cardiac resynchronization therapy 78 in patients with heart failure and ventricular conduction delay. J Am Coll Cardiol. 2002;39(12):2026-33.

19. Gasparini M, Lunati $M$, Santini M, Tritto $M$, Curnis A, Bocchiardo $M$, et al. Longterm survival in patients treated with cardiac resynchronization therapy: a 3- year follow-up study from the InSync/InSync ICD Italian Registry. Pacing and Clinical Electrophysiology. 2006;29(Suppl 2):S2-10. 